\title{
Extraction and Recycling of Battery Materials
}

\author{
CAMILLE FLEURIAULT, ${ }^{1,3}$ XIAOFEI GUAN,${ }^{2}$ and JOE GROGAN ${ }^{1}$ \\ 1.-Gopher Resource, Eagan, MN 55121, USA. 2.-School of Physical Science and Technology, \\ ShanghaiTech University, Shanghai 201210, China. 3.-e-mail: Camille.Fleuriault@ \\ GopherResource.com
}

Current battery recycling rates highly depend on the battery type and usage as well as available technologies. With a wide range of constraints on supply materials and stricter environmental standards, the necessity for not only expanding recycling abilities but also increasing performance is critical. Among the many types of battery available, this topic specifically covers lead acid and lithium ion chemistries. With the rise of electric vehicle, electric grid storage and electronics applications, spent lithium ion batteries (LIB) are quickly accumulating, and the recycling of the highly valuable elements such as lithium and cobalt can bring both economic and environmental benefits. While recycling solutions do exist and are employed in Europe, Asia and North America, the processing capacity for the expected surge is still too low. Lead acid battery (LAB) recycling benefits from a long history and a well-developed processing network across most continents. Yet, LAB recycling is subject to continuous optimization efforts because of increasingly stringent regulations on process discharge and emissions. In this special topic, nine featured publications discuss new findings in the fields of battery dismantling and separation, leaching and roasting optimization as well as electrochemical reduction.

In the first contribution, Pinegar and Smith investigate optimization of the upstream segment of the battery recycling process in "End-of-Life Lithium-Ion Battery Component Mechanical Liberation and Separation." Various mechanical and physical treatments processes were evaluated for improved LIB component separation and liberation of active electrode materials. The study demonstrates that shredding and vibration sieving followed by attrition milling is a promising, energyeffective LIB pre-processing route.

Xiaofei Guan is the $J O M$ advisor for the Recycling and Environmental Technologies Committee of the TMS Extraction \& Processing Division and the Light Metals Division; Camille Fleuriault and Joe Grogan are the JOM advisors for the Pyrometallurgy Committee of the TMS Extraction \& Processing Division. All were guest editors for the topic Extraction and Recycling of Battery Materials in this issue.
LIB breakage and separation also has many applications in the automation field. "Disassembly Automation for Recycling End-of-Life Lithium-Ion Pouch Cells" by $\mathrm{Li}$ et al. describes different prototypes for three steps of the LIB cathode recovery process: pouch trimming, housing removal and electrode sorting. While typically processed by destructive methods such as crushing, the authors introduce a new automated sequence which allows separation of cathode sheets, anode sheets, separators and aluminum film. This nondestructive process provides new opportunities for downstream treatment of the LIB. The publication also includes a concept verification with dismantling of dummy pouch cells.

"Biomass-Assisted Reductive Leaching in $\mathrm{H}_{2} \mathrm{SO}_{4}$ Medium for the Recovery of Valuable Metals from Spent Mixed-Type Lithium-Ion Batteries" by Chen et al. illustrates the viability and kinetic implications of using waste tea as reducing agent during sulfuric acid leaching of spent Li-ion cathodes. Under otherwise identical leaching conditions, the study shows the addition of waste tea allows an increase in the leaching efficiency of $\mathrm{Li}, \mathrm{Ni}, \mathrm{Mn}$ and Co. Presented data also provide optimal leaching conditions (namely concentration, leaching time and temperature) as well as a kinetic model suggesting that waste tea biomass constitutes a promising alternative to currently used reductants.

Recycling of LIB also involves pyrometallurgical processes. In "Sulfation Roasting Mechanism for Spent Lithium-Ion Battery Metal Oxides Under $\mathrm{SO}_{2}-\mathrm{O}_{2}$-Ar Atmosphere," Shi et al. develop a proof of concept for LIB processing via the direct sulfation roasting of synthetic $\mathrm{LiCoO}_{2}$ followed by water leaching. The study includes a comparison between experimental results and thermodynamic predictions as well as a thorough phase characterization of the process products. Findings of the study include recommendations for optimization of this new recovery path. 
In "Carbothermal Reduction of Spent Mobile Phones Batteries for the Recovery of Lithium, Cobalt, and Manganese Values," Pindar and Dhawan report a process for recycling discarded mixed mobile phone batteries incorporating indigenous reduction followed by leaching, magnetic separation and sulfuric acid leaching. The study shows that cobalt, lithium and manganese elements are successfully extracted from the cathode material compromising $\mathrm{LiCoO}_{2}$ and $\mathrm{LiMn}_{2} \mathrm{O}_{4}$.

"Preparation of Ternary Precursor Derived from Spent $\mathrm{LiNi}_{x} \mathrm{Co}_{y} \mathrm{Mn}_{1-x-y} \mathrm{O}_{2}$ Materials" by Liu et al. studies the regeneration of $\mathrm{Ni}_{x} \mathrm{Co}-\mathrm{Mn}_{1-x-y}(\mathrm{OH})_{2}$ precursors from spent LIB materials. This study combines hydro- and pyrometallurgical methods to recycle spent cathode materials by evaluating the feasibility of conducting reduction roasting, leaching and co-precipitation processes on crushed EOL components. The authors succeed in segregating $\mathrm{Li}$ in a carbonate phase while deporting $\mathrm{Ni}$, Co and $\mathrm{Mn}$ to a leachate suitable for precipitation. The end product, a resynthesized precursor, appears to be comparable to commercial equivalent and could have a direct application in manufacturing new cathodes.

Modeling plays a key part in the development of reliable, efficient extraction processes for battery materials. In "CFD-PBM Simulation and PIV Measurement of Liquid-Liquid Flow in a Continuous Stirring Settler," Guo et al. investigate optimization opportunities for widely used mixer settlers. Using computational fluid dynamics, velocimetry and a laboratory-scale continuous settler mixer, the authors analyze the droplet distribution patterns and droplet size when changing agitation speed of the mixer. Both experimental results and modeling showed that the mixing and settling performance can be accurately predicted and optimized using Ansys Fluent.

The second part of this topic is dedicated to environmentally friendly processes for LAB recycling. In "Clean Recycling Process for Lead Oxide Preparation from Spent Lead-Acid Battery Pastes Using Tartaric Acid-Sodium Tartrate as a Transforming Agent," Ouyang et al. present a novel desulfurization-calcination procedure. Sulfur removal of LAB paste is experimentally conducted using tartaric acid and sodium tartrate to produce a lead tartrate product. A calcination step then yields lead oxide powder. Upon microscopy analysis, the authors have found that the fine powder has potential for commercialization.

The final contribution on this topic also investigates opportunities for LAB paste recycling but this time via electrolytic processing. In "Preparation of
Metal Lead from Waste Lead Paste by Direct Electrochemical Reduction in $\mathrm{NH}_{3}-\mathrm{NH}_{4} \mathrm{Cl}$ Solution," Fan et al. present voltammetry experiments on synthetic lead paste in $\mathrm{NH}_{3}-\mathrm{NH}_{4} \mathrm{Cl}$ environment. The authors present the influence of scanning rate, temperature and electrolyte concentration on lead paste reduction.

The following contributions are published under the topic "Extraction and Recycling of Battery Materials" in the December 2019 issue (vol. 71, no. 12 ) of $J O M$ and can be accessed via the $J O M$ page at http://link.springer.com/journal/11837/71/12/page/1:

- "End-of-Life Lithium-Ion Battery Component Mechanical Liberation and Separation" by $\mathrm{H}$. Pinegar and Y. Smith.

- "Disassembly Automation for Recycling End-oflife Lithium-ion Pouch Cells" by L. Li, P. Zheng, T. Yang, R. Sturges, M. Ellis, and Z. Li.

- "Biomass-Assisted Reductive Leaching in $\mathrm{H}_{2} \mathrm{SO}_{4}$ Medium for the Recovery of Valuable Metals from Spent Mixed-Type Lithium-Ion Batteries" by Y. Chen, D. Chang, N. Liu, F. Hu, C. Peng, X. Zhou, J. He, Y. Jie, H. Wang, B. Wilson, and M. Lundstrom.

- "Sulfation Roasting Mechanism for Spent Lithium-Ion Battery Metal Oxides Under $\mathrm{SO}_{2-}$ $\mathrm{O}_{2}$-Ar Atmosphere" by J. Shi, C. Peng, M. Chen, Y. Li, H. Eric, L. Klemettinen, M. Lundstrom, P. Taskinen, and A. Jokilaakso.

- "Carbothermal Reduction of Spent Mobile Phones Batteries for the Recovery of Lithium, Cobalt, and Manganese Values" by S. Pindar and N. Dhawan.

- "Preparation of Ternary Precursor Derived from Spent $\mathrm{LiNi}_{x} \mathrm{Co}_{y} \mathrm{Mn}_{1-x-y} \mathrm{O}_{2}$ Materials" by P. Liu, L. Xiao, H. Chen, and H. Chen.

- "CFD-PBM Simulation and PIV Measurement of Liquid-Liquid Flow in a Continuous Stirring Settler" by X. Guo, T. Zhang, Q. Zhao, Z. Zhang, and S. Zhu.

- "Clean Recycling Process for Lead Oxide Preparation from Spent Lead-Acid Battery Pastes Using Tartaric Acid-Sodium Tartrate as a Transforming Agent" by Z. Ouyang, S. Liu, Y. $\mathrm{Hu}$, and $\mathrm{L}$. Ye.

- "Preparation of Metal Lead from Waste Lead Paste by Direct Electrochemical Reduction in $\mathrm{NH}_{3}-\mathrm{NH}_{4} \mathrm{Cl}$ Solution" by Y. Fan, Y. Liu, L. Niu, W. Zhang, and T. Zhang.

Publisher's Note Springer Nature remains neutral with regard to jurisdictional claims in published maps and institutional affiliations. 